MATEC Web of Conferences 11,01031 (2014)

DOI: $10.1051 /$ matecconf $/ 20141101031$

(C) Owned by the authors, published by EDP Sciences, 2014

\title{
Caractérisation de la corrosion d'acier par la méthode ultrasonore en mode de réflexion
}

\author{
Aziam Salma ${ }^{1}$, Nounah Hassan ${ }^{1}$, Aboudaoud Idris ${ }^{1}$, El abassi Dris ${ }^{1}$ \\ ${ }^{1}$ Université Ibn Zohr, Faculté des Sciences, Département de physique, Laboratoire de Métrologie et Traitement de \\ l'Information, Agadir, Maroc
}

\begin{abstract}
The ultrasonic method is widely used to detect and characterize internal defects, but also to measure the residual thickness in the presence of corrosive or erosive phenomena. In this work, we have exploited: the ultrasonic reflection technique using a transducer center frequency $5 \mathrm{MHz}$, which aims to deduct the ultrasonic parameters of samples, and tracing the reflection coefficient for each experimental sample. This work has demonstrated the reliability and the ability of ultrasonic techniques to show the influence of immersion time in a corrosive solution. The resulting corrosion on the corroded steel was analyzed by monitoring the evolution of ultrasonic parameters. We confirm some of the results found by other researchers ([1] (K. Niboucha, 2006) [2] (Benjamin L. Ervin, 2009) [3] (Vervisch-Isabelle Fortune, 2009) [4] (S. Sharma, 2010), the influence of the corrosion process of steels with nondestructive testing methods.
\end{abstract}

\begin{abstract}
Résumé. La méthode ultrasonore est très largement utilisée pour détecter et caractériser des défauts internes, mais aussi pour mesurer des épaisseurs résiduelles en présence de phénomènes corrosifs ou érosifs. Dans ce travail, Nous avons exploité : la technique ultrasonore en réflexion servant d'un transducteur de fréquence centrale $5 \mathrm{MHz}$, dont le but de déduire les paramètres ultrasoniques des échantillons, et du traçage du coefficient de réflexion expérimentale correspondant à chaque échantillon. Ce travail a mis en évidence la fiabilité et l'aptitude des techniques ultrasonores pour montrer l'influence de la durée d'immersion dans une solution corrosive. La corrosion résultante sur l'acier corrodé est analysée par le suivi de l'évolution des paramètres ultrasonores. Nous confirmons certains résultats trouvés par d'autres chercheurs ([1] (K. Niboucha, 2006); [2] (Benjamin L. Ervin, 2009); [3] (Vervisch-Fortuné Isabelle, 2009); [4] (S. Sharma, 2010), sur l'influence du processus de corrosion des aciers avec des méthodes de contrôle non destructif.
\end{abstract}

\section{Nomenclature :}

$\mathrm{e}:$ l'épaisseur de la plaque en $\mathrm{mm}$

L: la largeur de la plaque en $\mathrm{cm}$

$1:$ la longueur de la plaque en $\mathrm{cm}$

$\rho$ : la masse volumique en $\mathrm{Kg} / \mathrm{m}^{3}$

$\mathrm{t}:$ la durée d'immersion de la plaque dans $\mathrm{HCl}(5 \mathrm{~N})$

\section{Présentation des échantillons à caractériser}

Le tableau ci-dessous représente les échantillons des plaques d'aciers étudiées pendant ce travail.

\begin{tabular}{|l|l|l|l|l|l|}
\hline La plaque & $\begin{array}{l}\mathrm{e} \\
(\mathrm{mm})\end{array}$ & $\begin{array}{l}\mathrm{L} \\
(\mathrm{cm})\end{array}$ & $\begin{array}{l}1 \\
(\mathrm{~cm})\end{array}$ & $\rho$ & $\mathrm{t}$ \\
\hline Acier 1 & 2 & 7,71 & 10,01 & 7471,12 & $2 \mathrm{~h}$ \\
\hline Acier 2 & 3 & 9,91 & 10,33 & 7268,38 & $2 \mathrm{~h}$ \\
\hline Acier 3 & 3 & 7,62 & 8,78 & 7059,9 & $4 \mathrm{~h}$ \\
\hline Acier 4 & 2 & 7,96 & 7,98 & 7419,6 & $24 \mathrm{~h}$ \\
\hline $\begin{array}{l}\text { Acier } \\
\text { oxydé }\end{array}$ & 3 & 7,56 & 9,01 & 7083,056 & - \\
\hline $\begin{array}{l}\text { Acier } \\
\text { galvanisé1 }\end{array}$ & 3 & 9,93 & 10 & 7653,5 & - \\
\hline
\end{tabular}

\section{La technique ultrasonore par Réflexion}

This is an Open Access article distributed under the terms of the Creative Commons Attribution License 2.0, which permits unrestricted use, distribution, and reproduction in any medium, provided the original work is properly cited. 


\subsection{Description de dispositif expérimentale}

Dans cette méthode, le transducteur utilisé de fréquence centrale $5 \mathrm{MHz}$ fonctionne alternativement en émetteur et en récepteur. Le générateur d'impulsion excite électriquement le transducteur. Il insone sous incidence normale la cible. La pression acoustique rétrodiffusée est captée par le même transducteur. Le signal reçu transite par le même câble que le signal d'émission et arrive sur le connecteur T/R depuis lequel le signal d'émission a été envoyé. Grâce à une porte et à un système à retard intégrés au générateur d'impulsions, le signal reçu est visualisé en reliant par un câble coaxial le connecteur de signal de sortie à une voie de l'oscilloscope HP54600B de contrôle, synchronisé avec le générateur d'impulsions. L'oscilloscope est relié à l'ordinateur par un bus IEEE en vue de traitements du signal.

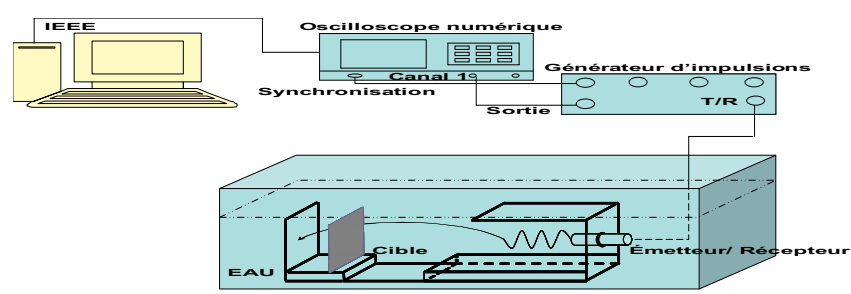

Fig.1. Schéma présentant le montage utilisé.

\subsection{Résultats expérimentaux}

\section{a. Technique de mesure de la vitesse en} utilisant le temps de vol

L'onde ultrasonore émise se propage à une vitesse qui dépend des caractéristiques de la plaque. Pour mesurer la vitesse de propagation d'une telle onde au sein de la plaque, on peut mesurer la durée entre le début de l'écho A1 et le début de l'écho A2. Cette durée correspond à un aller-retour de l'onde ultrasonore au sein de la plaque d'épaisseur $\mathrm{d}$.D'où, on en déduit la vitesse de propagation en appliquant la relation classique :

$$
V_{L}=\frac{2 d}{t_{v}}
$$

A partir du temps de vol $t_{v}$ de l'impulsion ultrasonore et connaissant l'épaisseur $d$ de la plaque, on remonte directement à la vitesse de phase longitudinale $\mathrm{V}_{\mathrm{L}}$.

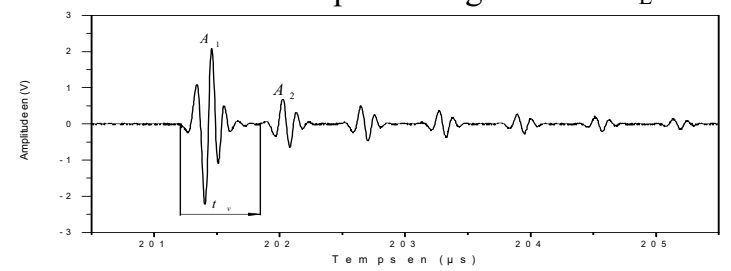

\section{b. Les résultats de l'étude expérimentale}
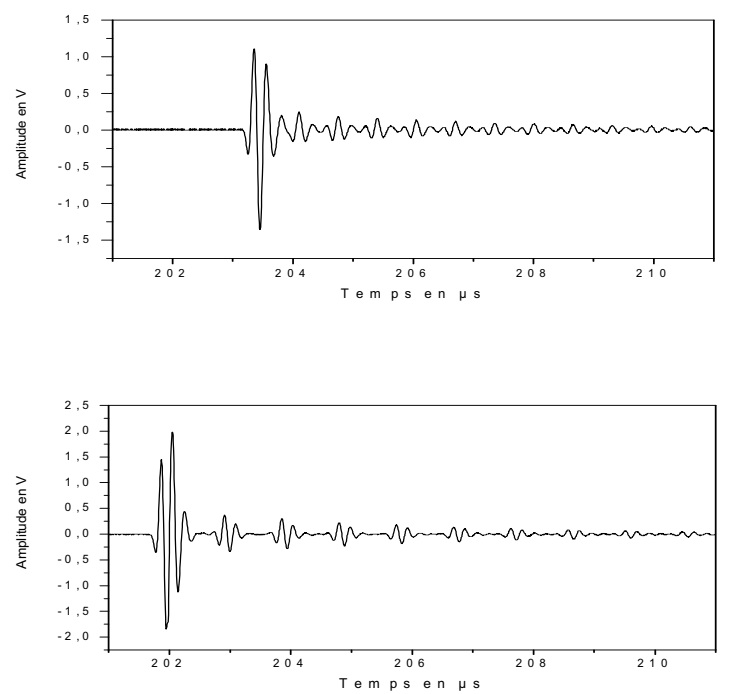

Fig.2. Signal temporel de rétrodiffusion par l'acier1 (haut), l'acier2 (bas)
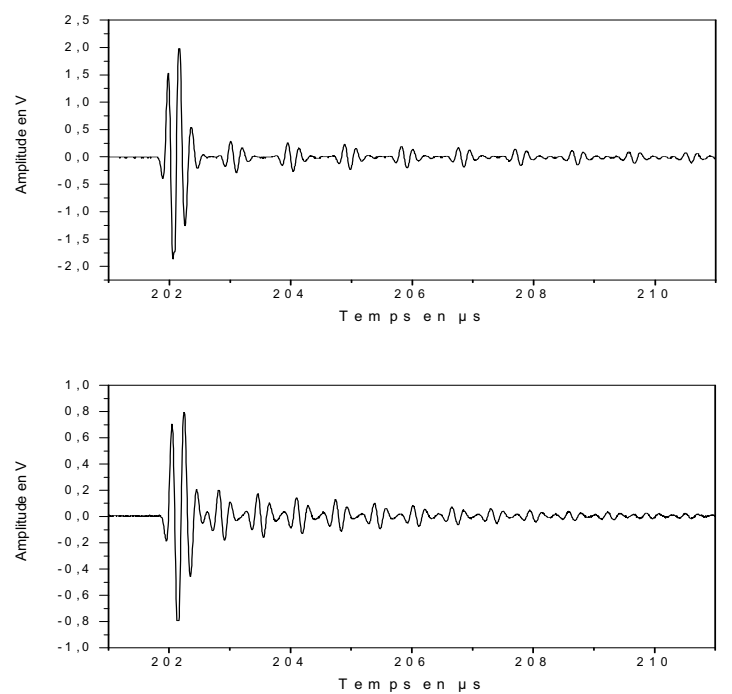

Fig.3. Signal temporel de rétrodiffusion par l'acier3 (haut), l'acier4 (bas).

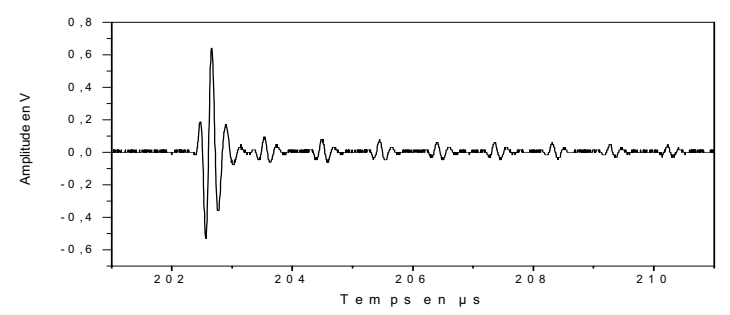

Fig.4. Signal temporel de rétrodiffusion par l'acier oxydé.

Nous avons mesuré le temps de vol tv pour les différentes plaques, et nous avons calculé leur vitesse longitudinale en appliquant la formule suivante :

$$
V_{L}=\frac{2 d}{t_{v}}
$$

Les résultats obtenus sont regroupés dans le tableau. 


\begin{tabular}{|l|l|l|}
\hline La plaque & Temps de vol & $\begin{array}{c}\text { Vitesse longitudinale } \\
(\mathrm{m} / \mathrm{s})\end{array}$ \\
\hline Acier 1 & $663,26 \mathrm{~ns}$ & 6030,81 \\
\hline Acier 2 & $980 \mathrm{~ns}$ & 6122,45 \\
\hline Acier 3 & $980 \mathrm{~ns}$ & 6122,45 \\
\hline Acier 4 & $800 \mathrm{~ns}$ & 5000 \\
\hline Acier oxydé & $920 \mathrm{~ns}$ & 6521,73 \\
\hline
\end{tabular}

Tableau récapitulatif de temps de vol et les vitesses longitudinales.

En analysant les résultats des mesures expérimentales obtenues par la méthode ultrasonore en réflexion mentionnés dans le tableau ci-dessus, nous déduisons l'existence d'une relation entre la durée d'immersion dans la solution corrosive et la vitesse longitudinale des ondes ultrasonores dans les différentes plaques d'acier corrodées. A partir des résultats illustrés dans le tableau ci-dessus, nous déduisons que plus la durée d'immersion de la plaque d'acier dans la solution corrosive est grande plus la vitesse longitudinale des ondes ultrasonore dans celle-ci diminue. Concernant les mesures du temps de vol, nous observons pour les plaques ayant une épaisseur identique et ayant une durée d'immersion largement différente par exemple la plaque d'acier $1(2 \mathrm{~h})$ et la plaque d'acier $4(24 \mathrm{~h})$ : le temps de vol dans la première plaque est inférieur à celui de la deuxième plaque. Pour les deux plaques d'acier 2et 3 ayant une épaisseur identique et une durée d'immersion qui n'est pas aussi bien large, nous observons que ces deux plaques ont un temps de vol identique. En comparant la plaque d'acier oxydée avec les plaques d'acier 2et 3 (ces trois plaques ont une épaisseur identique) : le temps de vol correspondant à la plaque oxydée est inférieur à celui correspondant aux plaques d'acier 2 et 3.

Nous pouvons expliquer ces résultats des mesures expérimentaux par le fait que l'effet de la corrosion sur une plaque d'acier consiste dont l'apparition d'un dépôt ou un résidus sous forme d'une couche dont l'épaisseur augmente suivant la durée d'immersion de la plaque dans la solution corrosive. L'augmentation de l'épaisseur de cette couche va influencer automatiquement sur le temps de vol. Ce dernier paramètre va augmenter suivant l'augmentation de l'épaisseur de la couche résultée de la corrosion. Par conséquent la vitesse longitudinale ultrasonore va diminuer car celle-ci est inversement proportionnelle au temps de vol. Nous avons mesuré un temps de vol identique et une vitesse longitudinale identique pour les plaques d'acier 2et 3 car elles ont une épaisseur identique. En effet ces deux plaques diffèrent dans la durée d'immersion dans la solution corrosive, la différence entre les deux plaques est de $2 \mathrm{~h}$ d'immersion. Cette différence apparait en conséquence dans l'épaisseur de la couche résultée de la corrosion et par conséquent dans le temps de vol correspondant. Pour atteindre la précision au niveau du temps de vol nous devrions travailler avec transducteur ultrasonore de haute fréquence par rapport à celle du transducteur utilisé (5 $\mathrm{MHz})$ durant ce travail. Avec cette fréquence $(5 \mathrm{MHz})$ nous ne pouvons pas détecter cette différence au niveau de l'épaisseur de la couche résultée de la corrosion. D'ailleurs dans les mesures expérimentales nous avons négligé l'épaisseur de cette couche car il est dans l'ordre des micromètres.

Ce résultat très important consiste dont le fait que l'épaisseur de la couche de corrosion augmente avec le temps d'immersion croissant.

\section{c. Le coefficient de réflexion}

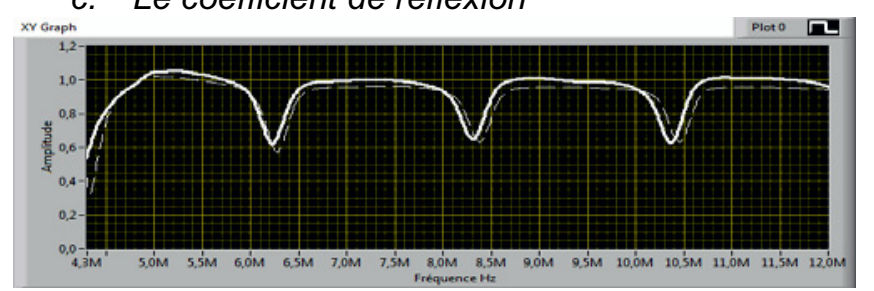

Fig.5. Le coefficient de réflexion d'acier 3 (pointillé) et acier 2 (continu)

Nous observons dans cette figure, qu'il n'y a pas une grande différence entre les coefficients de réflexion correspondant à ces deux plaques. Nous pouvons expliquer ce décalage au niveau de l'amplitude par la différence entre les deux plaques dont la durée d'immersion au sein de la solution corrosive. De plus, la différence au niveau de la durée d'immersion entre ces deux plaques est faible (environ 2 heures) ainsi le résultat montre clairement cette différence: l'amplitude du coefficient de réflexion de la plaque 2 est légèrement supérieur à celui de la plaque 3 .

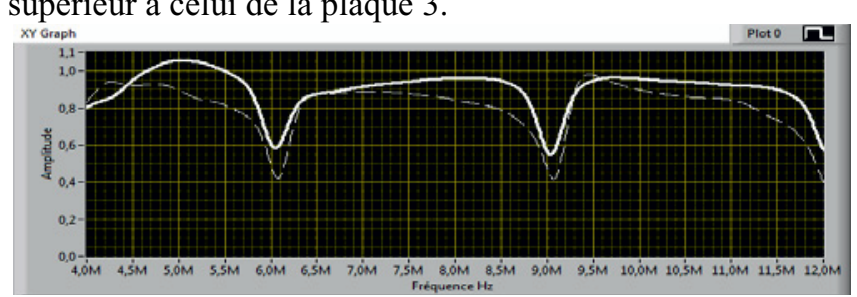

Fig. 6.Le coefficient de réflexion d'acier 1(continu) et d'acier 4 (pointillé).

La figure 6 signifie qu'il y a une grande différence entre les coefficients de réflexion correspondant à ces deux plaques au niveau de l'amplitude. Nous pouvons expliquer ce large écart au niveau de l'amplitude par la différence entre les deux plaques dont la durée d'immersion au sein de la solution corrosive. Ainsi le résultat montre clairement cette différence au niveau de la durée d'immersion entre ces deux plaques (environ 22 heures), qui est grande : l'amplitude du coefficient de réflexion de la plaque 1 est supérieure par rapport à celui de la plaque 4 .

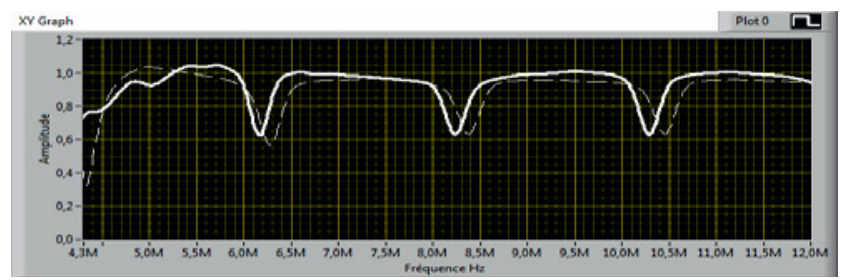

Fig. 7. Le coefficient de réflexion d'acier 3(pointillé) et acier oxydé (continu).

Nous remarquons qu'il y a un léger décalage au niveau de l'amplitude entre les coefficients de réflexion 
correspondant à ces deux plaques. Nous pouvons expliquer l'écart au niveau de l'amplitude par la différence du processus ou bien le mécanisme de la corrosion déclenchée par une solution corrosive et celui résulté sous l'effet d'oxydation naturel. Ainsi le résultat montre clairement cette différence: l'amplitude du coefficient de réflexion de la plaque 3 est inférieure par rapport à celui de la plaque oxydée.

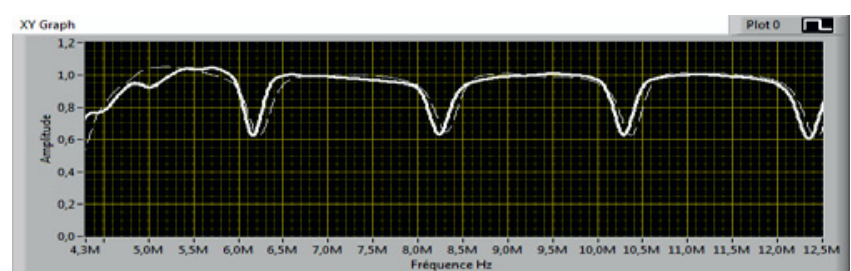

Fig. 8. Le coefficient de réflexion d'acier 2(pointillé) et acier oxydé (continu).

D'après La figure 8, on observe qu'il n'y a pas de différence significative au niveau de l'amplitude entre les coefficients de réflexion correspondant à ces deux plaques. Ce résultat montre que l'influence de la durée d'immersion joue un rôle très important au niveau de l'amplitude du coefficient de réflexion, plus la durée d'immersion est grande plus la différence au niveau de l'amplitude du coefficient de réflexion est importante.

\section{Conclusion}

Cette recherche a permis de démontrer l'effet de la durée d'immersion dans une solution corrosive sur les propriétés mécaniques des plaques d'acier corrodées. Nous avons exploité la technique ultrasonore en réflexion dont le but d'étudier l'effet de la corrosion sur les paramètres ultrasonores. Nous avons remarqué que l'augmentation du temps de vol suivant la durée d'immersion entraine la diminution de la vitesse longitudinale des ondes ultrasonores dans l'acier corrodée. Nous avons utilisé cette méthode pour mesurer le coefficient de réflexion correspondant à chaque plaque. En analysant les résultats, nous avons remarqué la diminution $\mathrm{du}$ coefficient de réflexion ultrasonore suivant la durée d'immersion. Ce résultat nous a permis de démontrer l'effet de la corrosion sur l'atténuation ultrasonore qui tient à augmenter si nous augmentons la durée d'immersion. Ce résultat nous montre l'efficacité de la technique ultrasonore en réflexion surtout si en exploitant les hautes fréquences car elle va nous permettre une haute résolution si nous voulons détecter l'épaisseur de la couche résultée de la corrosion.

\section{References}

1. Karima Niboucha, H. Houali, M. Zergoug, R. Halimi. Contrôle et caractérisation de la corrosion par CND pour l'inspection des canalisations enterrées, Dijon, France, (2006).

2. Benjamin L. Ervin, Daniel A. Kuchma, Jennifer T. Bernhard, and Henrique Reis. Monitoring corrosion of rebar embedded in mortar using high-frequency guided ultrasonic waves. Journal of engineering mechanics, Vol 135, No 1, pp.9-19, (2009).
3. Vervisch-Fortuné Isabelle, Sensibilité de la technique d'émission acoustique à la corrosion des armatures dans le béton. Thèse de doctorat, Toulouse, France, (2009).

4. Shruti Sharma and Abhijit Mukherjee, Longitudinal guided waves for monitoring chloride corrosion in reinforcing bars in concrete, Structural Health Monitoring, Vol 9(6): 555-13, (2010). 